\title{
Mode II fracture toughening and healing of composites using supramolecular polymer interlayers
}

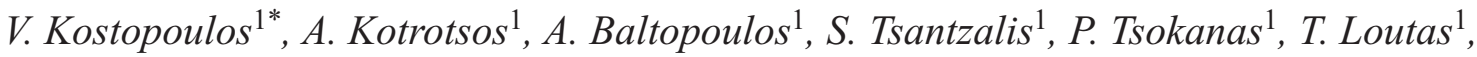 \\ A. W. Bosman ${ }^{2}$ \\ ${ }^{1}$ Applied Mechanics Laboratory, Department of Mechanical Engineering and Aeronautics, University of Patras, 26504 \\ Rio-Patras, Greece \\ ${ }^{2}$ SupraPolix BV, Horsten 1, 5612 AX Eindhoven, The Netherlands
}

Received 18 May 2016; accepted in revised form 25 June 2016

\begin{abstract}
This study focuses on the transfer of the healing functionality of supramolecular polymers (SP) to fibre reinforced composites through interleaving. SPs exhibiting self-healing based on hydrogen bonds were formed into films and were successfully incorporated into carbon fibre composites. The effect of the SP interleaves on in-plane fracture toughness and the subsequent healing capability of the hybrid composites were investigated under mode II fracture loading. The fracture toughness showed considerable increase since the maximum load $\left(P_{\max }\right)$ of the hybrid composite approximately doubled, and consequently the mode II interlaminar fracture toughness energy $\left(G_{\text {IIC }}\right)$ exhibited an increase reaching nearly $100 \%$ compared to the reference composite. The healing component was activated using external heat. $P_{\max }$ and $G_{\mathrm{IIC}}$ recovery after activation were measured, exhibiting a healing efficiency after the first healing cycle close to $85 \%$ for Pmax and $100 \%$ for $G_{\text {IIC }}$, eventually dropping to $80 \%$ for $P_{\max }$ while $G_{\text {IIC }}$ was retained around $100 \%$ even after the fourth healing cycle. Acoustic Emission activity during the tests was monitored and was found to be strongly reduced due to the presence of the SP.
\end{abstract}

Keywords: smart polymers, CFRPs, supramolecular polymers, fracture toughness, self-healing

\section{Introduction}

High performance structural composites are year by year replacing metals in load-critical structures of aircrafts and other industrial applications. This tendency is clearly driven by the need for structural materials with superior mechanical properties, low specific weight and corrosion resistance. On the other hand, during their service life, multi-layered composites are prone to delaminations, as a result of out of plane loads. The presence of delaminations dramatically decreases their residual compression properties and also affects other in-plane properties of the composite. Additionally, polymer matrix composites are sensitive to microcracking formation within the polymer phase due to service-induced loading or even during the manufacturing. Under fatigue loading conditions that a real-world structure experiences on its service life, the initially minor microcracks are linked together to create significantly larger cracks. Cracks and delaminations work together to result in remarkable suppression of the load-bearing capacity of the composite part, even with minimal visual indication of damage being present.

The interlaminar fracture toughness is the key-characteristic in initiation and propagation of delaminations in fibre reinforced polymer (FRP) composites. A constant concern of various researchers has been the enhancement of the fracture behavior of high performance structural composites, especially carbon fibre reinforced polymers (CFRP). In this direc-

$\overline{{ }^{*} \text { Corresponding author, e-mail: kostopoulos@mech.upatras.gr }}$ (C) BME-PT 
tion, a series of approaches has been proposed including thermoplastic interleaves, micro- and nanoparticles, other microfibres, z-pins etc [1-5]. However, the most common and widespread approach is the interleaving. The incorporation of very thin layers can be a feasible way to enhance the interlaminar properties without compromising other mechanical properties. For example, Magniez et al. [6] succeeded in the toughening of the CFRPs by interleaving a thin layer $(20 \mu \mathrm{m})$ of electrospun poly(hydroxyether of bisphenol A). This type of enhancement improved the fracture toughness in mode I and mode II by up to 150 and $30 \%$ respectively. Aksoy and Carlsson [7] achieved toughening of graphite/epoxy composites using thermoplastic and thermoset interleaves with various thicknesses. Both types of interleaves enhanced the interlaminar fracture toughness to a great extent, although thermoplastic interleaves were more effective than the thermoset interleaves because of the larger energy-absorbing capability of the thermoplastic polymer. Molnár et al. [8] investigated the interleaving effect of polyacrylonitrile electrospun mats on interlaminar shear stress and impact tests of unidirectional and woven CFRPs. It was shown that the interlaminar shear strength of the woven and unidirectional CFRPs was enhanced at the rate of 7 and $11 \%$ respectively. In the case of impact tests carried out on woven CFRPs the absorbed energy was increased at the rate of $64 \%$ compared to the neat ones. Drakonakis et al. [9] investigated the role of the host matrix and the micro-particle interleaving effect to the multilayer structures. From the three different matrices which were utilized, it was shown that the dicyanate was the toughest epoxy while the mode II fracture characteristics were considerably increased with the incorporation of Nylon 6 micro-particles.

Conventional repair techniques of composites are expensive, require extensive human work, and cannot repair defects deep inside the material. Thus, utilization of composites in human safety critical components has always to be accompanied with damage diagnostic tools. It is also of note that conventional damage detection equipment is in some cases not able to detect tiny defects deep inside the material. However, these defects could rapidly propagate between two periodical inspections and lead the composite part to catastrophic failure. This challenging situation acted as an inspiration for the seeking of new repair methods; cheaper and applicable at the early stages of damage evolution. With an aim to address some principal weak points of conventional repair techniques, an emerging approach called 'selfhealing polymers' [10] has been proposed but not yet been applied to commercial composites. This smart technology aims to in-situ and autonomously repair damage and thus to lead to extension of the effective life-span of composite structures. Self-healing composites promise to mitigate the importance for detecting damage and to reduce the frequency of scheduled inspections.

An alternative approach to the 'conventional' damage tolerance design philosophy is to provide a material with the ability to self-repair. To this direction, scientists have drawn inspirations by the ability of the biological systems to repair external wounds in order to develop similar repair systems within the material. Two main ways for implementing the healing functionality within a material have been reported over the past two decades: a) the extrinsic one, e.g. $[11,12]$, where the healing agent is liquid and stored in advance within the material, and b) the intrinsic one, e.g. [13, 14], where the inherently thermally reversible bonds of the material create the healing effect under external heating.

However a more effective way to address the longstanding problem of weak through-the-thickness strength of structural composites is probably the development of new composites that combine high interlaminar fracture toughness with the ability to selfheal. For example, Wang et al. [15] investigated the combined self-healing and toughening performance of CFRP interleaved with patches of two thermoplastics. The hybrid composite had enhanced mode I but reduced interlaminar shear properties while reasonable mechanical property recovery after healing was achieved via both thermoplastics in both loading cases. In addition, Yang et al. [16] proposed a new type of self-healing composite material containing a three-dimensional thermoplastic (EMAA) fibre system created by stitching. The hybrid composite was tougher than the reference and high recovery of the mode I fracture characteristics was achieved after multiple healing cycles. Very recently, Kotrotsos et al. [17] proposed a hybrid composite with a matrix modified with thermoplastic micro-particles. The com- 
posite had enhanced mode II fracture toughness and was able to significantly restore the mode II fracture characteristics after healing.

A more promising approach for self-healing composites might be found by merging reversible bonds into epoxide networks, since this approach allows the healing to be unlimited as no chemicals are consumed. A new technology that could be beneficial for self-healing in composites has been built on supramolecular polymers (SP) [18-20]. Especially those based on reversible hydrogen bonding arrays show great promise for self-healing materials [21-24], since these materials can typically withstand multiple healing cycles without substantial loss of performance, as a consequence of the highly directional and fully reversible non-covalent interactions present within the polymer matrix. In this study, we have employed the ureiodypyrimidone hydrogen bonding unit (UPy) as developed by Sijbesma et al. [25] because of its strong self-association, its synthetic accessibility, and the highly dynamic nature of low $T_{\mathrm{g}}$-polymers comprising the UPy [26]. Most interestingly, UPy-polymers have recently been shown to give unprecedented toughening in polybutadiene based interpenetrating networks [27]. In recent investigation, Zhang et al. [28] discussed the state of our understanding about ionomers and the historical applications of these nanostructured polymers. It was also discussed the modern methods for synthesizing new ionomers and described a number of relatively new applications for ionomers and the potential use of these materials in contemporary technologies, including, shape memory and self-healing materials and supramolecular polymer systems.

The scope of the present work is the use of flexible SP comprising UPy-moieties as interleaves into conventional CFRPs in order to enhance the fracture properties of these hybrid composites and to take advantage of the SP's healing capability. This study is presenting an overview of the role of the SP in the fracture behavior and the repeatable ability to heal the cracks into CFRPs. It is complementing and extending the work of study [29] where mode I fracture was investigated. Here, reference as well as the hybrid composites were subjected to mode II interlaminar fracture toughness tests and compared. After the fracture, the hybrid composites were subjected to heating under controlled loading in order to activate the SP interleave and the cracks to be healed. The healing process was repeated up to four times. Finally acoustic emission (AE) monitoring was implemented during the testing. Recordings led to better understanding of the involved failure mechanisms as well as some conclusions regarding the healing mechanism.

\section{Experimental}

\subsection{Materials}

The composite materials which were used in this study were fabricated from UD carbon fibre-epoxy pre-preg CE-1007 150-38. The pre-preg tape was supplied by SGL Group, Germany. The SP was developed and supplied by Suprapolix. The supplied material (batch identification code: SPSH01) is based on a low $T_{\mathrm{g}}\left(-66^{\circ} \mathrm{C}\right)$ polymer modified with UPymoieties, and was chosen to play the toughening and healing role as an interleaf in the present study. This polymer owes its mechanical properties to the reversible, non-covalent interactions, such as hydrogen bonding, between the macromolecules.

\subsection{Preparation of SP interleaf and composite manufacturing}

The as-received polymer piece was converted into a thin film by a two-step heating/pressing treatment using a hot press machine [29]. Firstly, the bulk SP block was pressed under $5 \mathrm{kN}$ at $90^{\circ} \mathrm{C}$ for $30 \mathrm{~min}$. Then, heating was stopped and the SP material was left under $25 \mathrm{kN}$ mechanical load overnight to cooldown and reach its final form. Consequently, a thin film of SP was obtained. The thickness of the film was measured to be approximately $120 \mu \mathrm{m}$ with no significant thickness variations, using a digital caliper. Finally, the SP film was cut into $20 \mathrm{~mm}$ wide strip using a conventional film cutter. The selected strip's width, based on experience ensures that the crack propagation would not overcome the healing agent area during the mode II experiments. The melting temperature $\left(T_{\mathrm{m}}\right)$ of the SP material was measured to be $77^{\circ} \mathrm{C}$, using the Perkin-Elmer DSC 8500 differential scanning calorimeter. The DSC samples were heated from ambient temperature to $150^{\circ} \mathrm{C}$ at a rate of $5^{\circ} \mathrm{C} / \mathrm{min}$. Above this temperature, the UPy-UPy hydrogen bonding interactions start to diminish significantly and thereby the interactions between adjacent polymer chains of the material [26]. 
Two UD laminated plates made of 22 pre-preg layers were manufactured for the needs of the current study; the reference laminate and the hybrid laminate containing the SP interleaf on the mid-plane, both appropriate for deriving mode II interlaminar fracture specimens. Figure 1 shows schematically the plate configuration with respect to the fracture pre-crack and the healing agent location. During the manufacturing process two $13 \mu \mathrm{m}$-thick sheets of polytetrafluoroethylene (PTFE) film were placed in the mid-thickness plane of both laminates as shown in Figure 1 to act as initial pre-crack according to the request of the interlaminar fracture test. In the case of the hybrid laminate, a SP strip was carefully placed on the mid-plane as shown in Figure 1b, 1c. Following the lay-up, the laminates were vacuum bagged and cured in autoclave for $2 \mathrm{~h}$ at $130^{\circ} \mathrm{C}$ under 6 bar pressure, according to the pre-preg supplier guidelines. The dimensions of the plates were $300 \mathrm{~mm} \times 150 \mathrm{~mm} \times 3 \mathrm{~mm}$. Ultrasonic $\mathrm{C}$-scan inspection was performed on the produced plates. A Physical Acoustics Corporation (PAC) UT C-Scan system was used with a $5 \mathrm{MHz}$ transducer. Five mode I (double cantilever beam, DCB) samples were cut from both the reference and the hybrid plate.

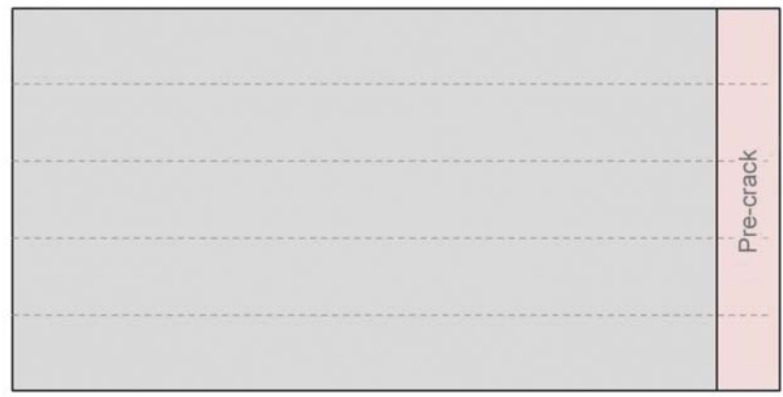

a)

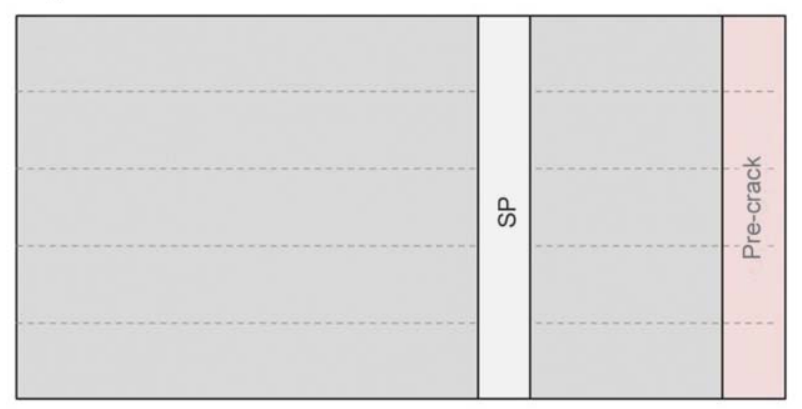

b)

\subsection{Mechanical testing and $\mathrm{AE}$ monitoring}

Quasi-static interlaminar fracture toughness tests were performed at a $25 \mathrm{kN}$ Instron Universal testing machine at room temperature conditions. Two aluminium tabs were glued on the DCB specimen outer surfaces (Figure 2a) using a two-component epoxy adhesive in order to bear the peel forces from the load cell. Both the reference and the hybrid DCB specimens were tested in mode I according to AITM 1.0005 [30] standard of Airbus defence and space, to create a natural sharp pre-crack. After the testing, the fractured samples were cut into mode II (ENF) specimens according to AITM 1.0006 [31] standard of Airbus. ENF specimen dimensions and experimental set-up are both illustrated in Figure 2b, 2c. The pre-cracked ENF test specimens were loaded in 3 -point bending, at a cross-head speed of $1 \mathrm{~mm} / \mathrm{min}$. Five replicates were tested for each fracture toughness assessment. The $G_{\text {IIC }}$ was determined using the Areas method [31], and calculated using the Equation (1):

$G_{\text {IIC }}=\frac{9 P a^{2} d}{2 w\left(\frac{1}{4} L^{3}+3 a^{3}\right)}\left[\frac{\mathrm{kJ}}{\mathrm{m}^{2}}\right]$

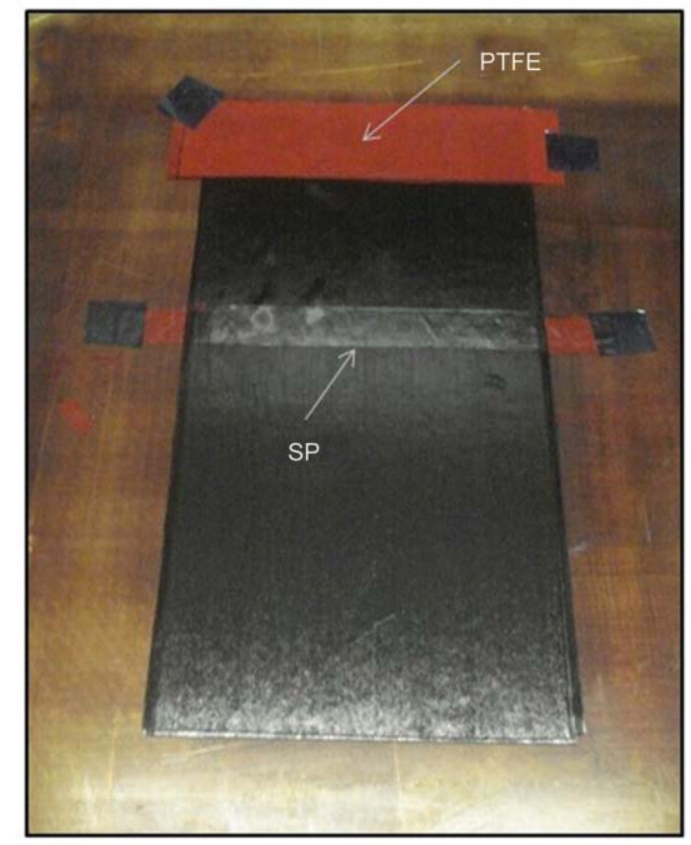

c)

Figure 1. Schematic representation of the mid-plane of (a) the reference plate and (b) the SP containing hybrid plate. (c) Photograph of the mid-plane of the hybrid plate, where a SP strip was carefully positioned, together with the PTFE film. 


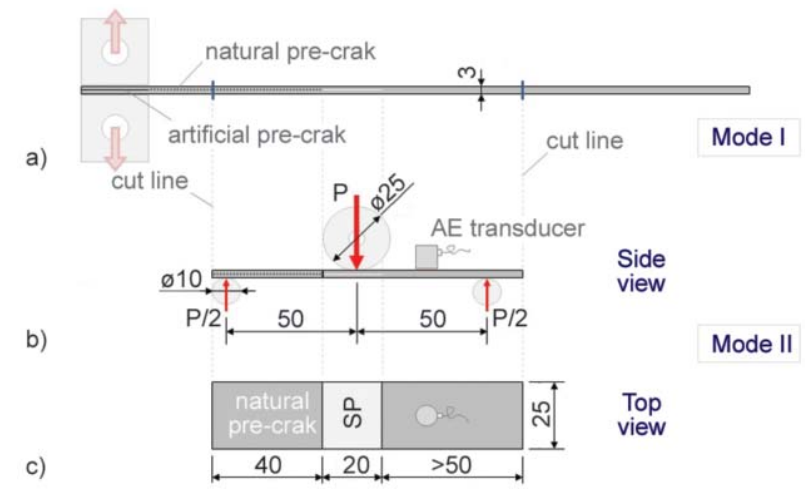

Figure 2. Schematic depiction of the hybrid ENF test specimen configuration as derived from DCB specimen as well as the topology of the AE transducer. (a) DCB specimen, (b) Side view of the ENF specimen, (c) Top view of the ENF specimen. Dimensions in $\mathrm{mm}$.

where $d$ is the cross-head displacement at crack delamination onset, $P$ is the critical load to start the crack, $a$ is the initial crack length (from the support point to the end of the crack), $w$ is the width of the specimen and $L$ is the span length $(L=100 \mathrm{~mm})$.

Based on Equation (1), the crack length $(a)$ has to be known in order the $G_{\text {IIC }}$ value to be calculated. For brittle materials the moment of unstable crack growth can be clearly determined; $P_{\max }$ is attributed to the critical load at the onset of crack growth. On the other hand, for hybrid materials containing ductile interleaves in which the crack is growing through the ductile interleaved layer [29], the crack initiation occurs long before $P_{\max }$. In addition, in mode II loading of the pre-cracked specimen the crack tends to close which hinder a clear visualization of its tip. Thus, mode II fracture characterization of CFRPs with a ductile interlayer remains a major challenge including uncertainties on the results. Based on these, whereas for the reference case CFRPs the $G_{\text {IIC }}$ calculations were extracted directly from the $P-d$ data without any concern, for the hybrid CFRPs the monitoring of the crack growth using high resolution digital camera system was carried out. It is suggested that the crack initiation point of the hybrid CFRPs corresponds to the onset of the visual deviation of linearity in $P-d$ curves. In addition, a vertical line with pencil was marked at the end of the starter film, as shown in Figure 2b. It is suggested that the separation of the line into two lines corresponds to the crack initiation.
In addition, during the tests the AE activity of the samples was monitored. AE is an ideally suited nondestructive technique for the on-line monitoring of the crack propagation. In this test campaign $\mathrm{AE}$ was utilized to support the extraction of useful conclusions, regarding the damage mechanisms activated during the mechanical experiments and investigate any changes due to the presence of the healing agent in the system. An AE sensor was mounted on the specimens' surface as shown in Figure 2b, 2c. The sensor type is wideband WD 100-900 kHz manufactured by PAC, USA. The transducer was attached on the specimens' surface using a suitable glycerinebased coupling agent. AE signal acquisition was performed via a four channel 16 bit PCI/DSP-4 by PAC data acquisition system. Pre-amplification of $40 \mathrm{~dB}$ and band-bass filtering of $20-1200 \mathrm{kHz}$ was performed using general purpose voltage preamplifiers with $0 / 20 / 40 \mathrm{~dB}$ variable gain (2/4/6-AST Auto Sensor Testing Preamplifiers by PAC). A threshold of $40 \mathrm{~dB}$ was chosen and the timing parameters were set at Peak Definition Time $(P D T)=50 \mu$ s, Hit Definition Time $(H D T)=100 \mu$ s and Hit Lockout Time $(\mathrm{HLT})=300 \mu \mathrm{s}$. HDT is defined as the maximum duration time of a signal to avoid the monitoring of its reflections. HLT is the waiting time of the system after the last threshold overcoming, to start the new recording. PDT identifies the maximum time between the peaks of two consecutive signals.

The study is deployed in two levels. The first level covers the assessment of the performance change due to the introduction of the healing agent in the reference CFRP system. It is possible that the approach to introduce the healing functionality may jeopardize the load-bearing capacity of the composite. This change in performance is commonly referred to as the knock-down effect. The second level of this study deals with the healing functionality of the composite system and its performance. Essentially, once the healing agent has been incorporated, the system is expected to have a healing functionality; meaning the capability to heal damage in the form of cracks (intrinsic or externally activated). The extent to which this functionality delivers its purposes is assessed in the second level of this work. Details on the procedure are given in the next paragraph. 


\subsection{Healing procedure and healing efficiency calculations}

After the testing procedure, the composites were subjected to a simple healing cycle. The healing cycle activated the healing agent in order to bridge the created crack and heal the system to recover its mechanical performance. The cycle comprised a $15 \mathrm{~min}$ dwell at $100^{\circ} \mathrm{C}$ under loading of $1 \mathrm{kN}$ using a hot press machine. The aforementioned healing characteristics were chosen in order to be sure that the healing agent would flow along the crack area. The samples were then left to cool-down to room temperature. After the healing process, the samples were retested using the same configurations and procedures described previously. The testing-healing process was repeated until the specimens had received 5 tests and 4 healing events. Reference composites were tested on their original state (un-healed). The calculations of the healing efficiency (H.E.) of the estimated system were based on Equation (2):

H.E. $=\frac{a_{\text {healed }}}{a_{\text {hybrid }}} \cdot 100 \%$

where $a$ is the property under examination (i.e., $P_{\max }$ or $\left.G_{\text {IIC }}\right), a_{\text {healed }}$ refers to the value of the property after healing and $a_{\text {hybrid }}$ refers to the property before healing. The H.E. value refers only to the composites with healing functionality.

\section{Results and discussion}

\subsection{Production and quality issues of composites with healing functionality}

Forming the SP material in film form was straightforward as it was easy to process it using processes similar to those used in thermoplastics forming. The handling of the SP film did not create any special concerns. At room temperature the film was stable and did not exhibit tackiness. During composite processing it did not create any problem. The tackiness of the pre-preg was sufficient for holding the film in position. The incorporation of the SP film in the midplane of the hybrid laminate did not appear to have a significant effect on the thicknesses of the samples. C-scan images confirmed CFRP plates' quality and showed the absence of manufacturing induced porosity or delaminations, as can be seen in Figure 3. In both plates the rectangular-shaped PTFE strip, which was positioned in the mid-plane of the laminate is clearly distinguished. Additionally, Figure $3 \mathrm{~b}$ shows clearly the position of the SP film insert, which was incorporated in the mid-plane of the hybrid laminate. The fiber volume fraction of all manufactured plates was calculated to be close to $60 \%$.

\subsection{Mode II fracture toughness: Reference vs. hybrid CFRP}

The introduction of the healing agent in the CFRP architecture is expected to have an impact on the material performance. At this part of the work the impact on mode II fracture toughness is assessed. The mechanical response of the reference and the hybrid CFRP to mode II fracture loading in combination with $\mathrm{AE}$ monitoring and crack evolution camera recording was studied.

Typical load-displacement $(P-d)$ curves for the reference and the hybrid plate are given in Figure 4a. All specimens within each set showed a similar behavior. The average values and standard deviation for the two material sets are shown in Figure 4b. As previously mentioned, the $P-d$ behavior of the two material sets differs. For the reference material, the applied load increases linearly until the onset of the pre-crack propagation where the load drops abruptly. On the other hand, for the hybrid CFRP the initially linear $P-d$ relation is followed by a distinguishable deviation from linearity and much latter the load drop appears. Whereas the load drop for the reference case is attributed to the crack evolution onset, for the hy-

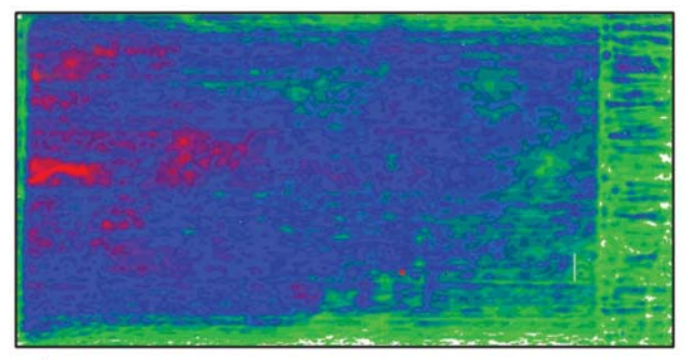

a)

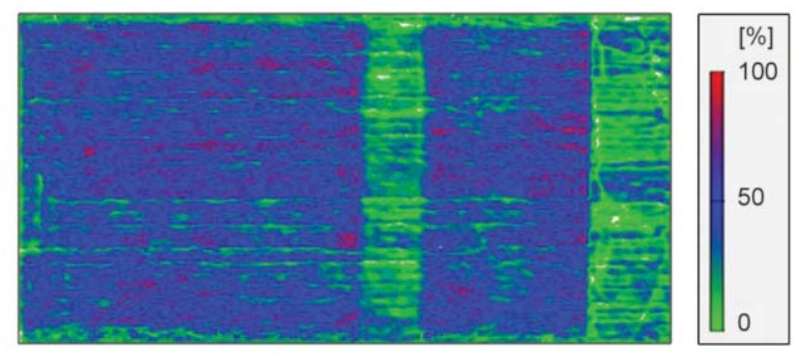

b)

Figure 3. C-scan inspection image of the produced plates: (a) Reference CFRP, (b) Hybrid CFRP 
brid CFRP the $P_{\max }$ indicates the load bearing capacity of the composite before undergoing major damages. Interestingly, the hybrid laminate exhibits a significantly higher $P_{\max }$ and respective displacement. As seen in the Figure $4 \mathrm{a}, P_{\max }$ is more than double that of the reference CFRP ( $120 \%$ increase) while the displacement at failure is approximately 4 times larger than the reference. From the recorded mechanical data the $G_{\text {IIC }}$ was calculated. For the calculation, the displacement $(d)$ at crack propagation onset is needed to be known. The crack initiation points for the reference and the hybrid CFRP, determined as described in section 2.3, are marked in Figure 4a. The snapshot of Figure 4a corresponds to the marked crack initiation point for the hybrid specimen. In this snapshot a slight separation of the pencil line into two lines is observed. The $G_{\text {IIC }}$ for both material sets was calculated using in Equation $(1)$ the $(P, d)$ values that correspond to the indicated points of the curve. The average values and standard deviation are shown in Figure $4 \mathrm{~b}$. The $G_{\text {IIC }}$ values for the hybrid CFRP exhibit an increase of $100 \%$ which is related both to the slightly higher corresponding load $(P)$ recorded but also to the displacement $(d)$ at that instance. Nevertheless, this important toughening takes place at a cost of the laminate bending stiffness; a $40 \%$ decrease at the linear portion of the curve between the reference and the hybrid CFRP is observed.

AE was recorded during the tests for both materials to support the investigation and are summarized in Figure 5. The AE characteristics in terms of cumulative AE hits and energy were calculated at the thresh-

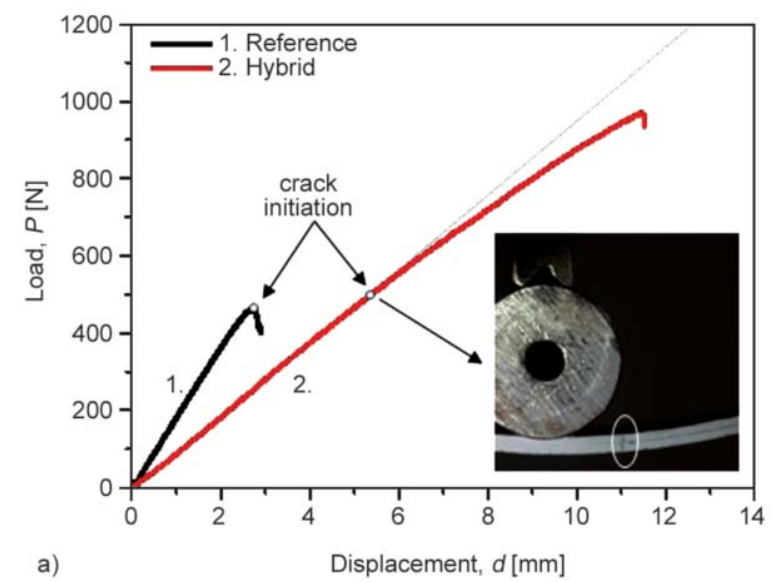

old of crack initiation (i.e., $P_{\text {ini }}$ ) for both types of samples as well as at the $P_{\max }$ point for the hybrid samples. It is important to highlight that the brittle nature of the reference composite made the $P_{\text {ini }}$ and $P_{\max }$ points to coincide while in the case of the hybrid ductile samples these points differ significantly, as shown in Figure 4a. It is evident in Figure 5 that the AE activity is significantly less in the hybrid case despite the fact that the test lasts much longer and the specimen experiences much higher loading and displacement. A similar behavior was observed in a previous work [29] in which the AE characteristics were strongly reduced during mode I experiments of hybrid CFRPs containing the same material interleaf. For the hybrid samples the AE activity is much higher at the fracture point in comparison to the point in which the crack initiates as anticipated. The AE activity almost doubled for both the AE characteristics (energy and hits) after the crack initiation point. Apparently, the viscoelastic SP interleaf plays a crucial

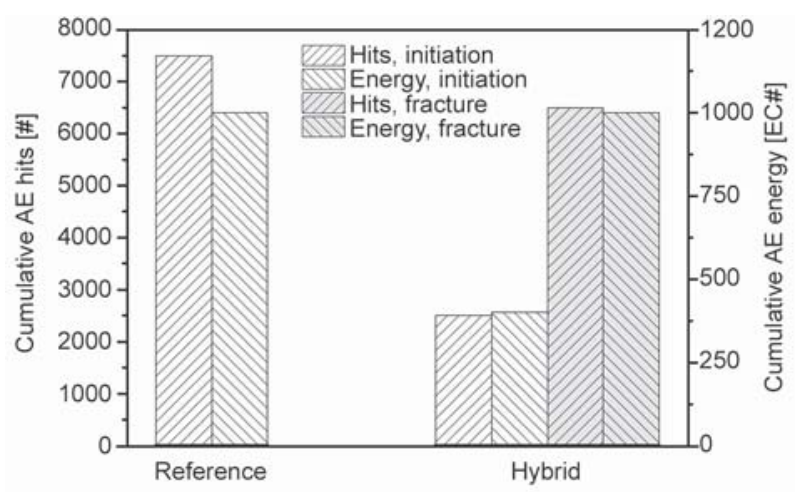

Figure 5. AE activity during mode II interlaminar fracture toughness tests of the reference and the hybrid CFRP at crack initiation and at fracture point

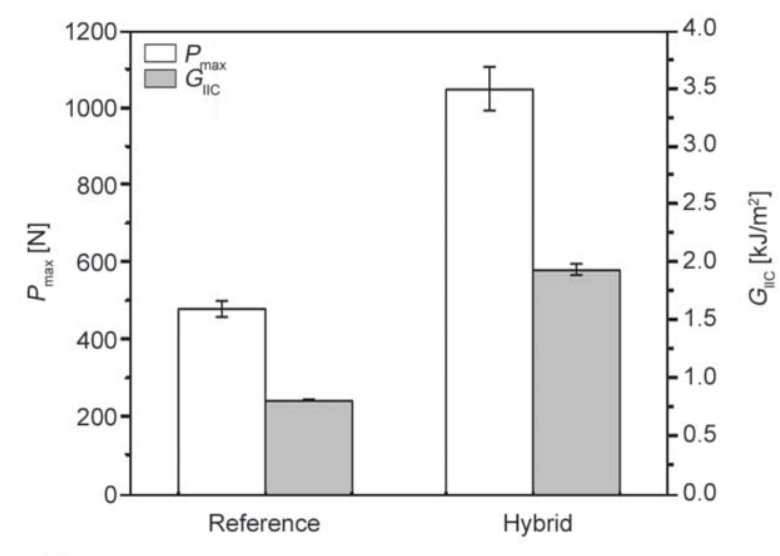

b)

Figure 4. (a) Mode II interlaminar fracture toughness test: representative load-displacement curves for the reference and hybrid CFRPs. (b) Comparison between the reference and the hybrid CFRP, for the maximum load $\left(P_{\max }\right)$ and the mode II interlaminar fracture toughness energy $\left(G_{\mathrm{IIC}}\right)$ 
role in suppressing damage mechanisms otherwise activated at much lower loads/displacements as in the reference case composite material. The macroscopic bending stiffness of the hybrid composite decreases as a knock-down effect but in return the SP interleaf offers extra resistance to the crack initiation as well as larger capacity of strain energy storage (much larger displacements).

As post-tests inspection revealed, the in-plane crack propagated through the SP interleaf rather than along the SP-epoxy interface. This observation indicates that the interface of the interleaf to the host matrix is stronger than the properties of the interleaf, thus guiding the crack to a lower energy path through the interleaf [29]. This behavior essentially maximizes the effectiveness of the interleaf and explains the higher resistance to crack propagation. In addition, high displacements are recorded while the load increased steadily. This can be related to the SP's ductile behavior in combination with its strength. Even when the crack initiated, the presence of the SP interleaf arrested the crack and prevented propagation due to the developed bridging [29] within the crack sides. This results in a lowering of the stress at the crack tip, and consequently in an increased mode II interlaminar fracture toughness. As a matter of fact, similar findings have been reported in by Aksoy and Carlsson where thermoplastics were investigated for interleave toughening. The high deformation of the interleaf resulted in the creation of 'shear-lips'. The deformation mechanism of the interleaf is believed to be similar to the formation of 'shear lips' in mode II fracture of ductile thermoplastic polymers [7]. The same toughening mechanism was also observed and analyzed in detail in the preceding work [29] where support from optical microscopy micrographs from both the cross-section and the fractured surfaces was provided. In Groleau et al. [33] investigation a similar mechanism is described in which the interlayered nylon particles played the role of toughening agent into the composite while exhibited plastic deformation and bridging. During the bridging process the particles absorbed energy through extensive plastic deformation and thus the $G_{\text {IIC }}$ value was increased.

As mentioned earlier, the flexural stiffness of the hybrid CFRP is lower than that of the reference material. The interleaf has lower stiffness than the UD composite plies and thus results in reducing the equivalent global stiffness of the CFRP plate. Following classical lamination theory this effect, affected by the supramolecular polymer's mechanical properties and the thickness of the interleaf, can be quantified. This is confirmed in fact by studies where thermoplastic interleaves are used to design composites with controllable stiffness [32].

SP provide a unique combination of properties as a material system. Many similarities exist between SP and elastomers, in terms of mechanical behavior, as well as with thermoplastics, primarily in terms of handling and processing. This gives rise to assess SP as engineering materials in general. Thus, on top of the healing functionality SP have repeatedly exhibited on polymer level [33] and which is later on fibre reinforced composite level, SP offer a complementary option in material selection which can deliver improved in-plane fracture toughness as described earlier based on the collected experimental data.

The results from the first study level clearly show that the presence of the supramolecular interleave in the composite resulted in a considerable increase of the mode II interlaminar fracture toughness characteristics. On the physical side, it is believed that the mechanisms delivering this increase in the exhibited fracture toughness are related both to the SP-epoxy interface as well as the structural properties of the interleaf itself. Thus, it is obvious that the fracture toughness characteristics of the composite have been enhanced significantly by the introduction of the SP interleaf. On the contrary, the performance of the CFRP is greatly enhanced. It can be concluded that, regardless of the healing functionality performance, this finding is extremely positive and of great interest.

\subsection{Assessment of healing functionality of composites}

The healing functionality of these supramolecular interleaves into CFRP composites was assessed by subjecting the samples to a healing cycle consisting of heating and compression as described in section 2.4. Indeed, a large recovery of the interlaminar characteristics $\left(P_{\max }\right.$ and $\left.G_{\text {IIC }}\right)$ during the repeated mode II experiments was observed when the fractured samples had been subjected to multiply healing cycles. 
In Figure $6 a$ the recorded load-displacement data are shown for a representative hybrid CFRP specimen. There are five curves; one for the initial test (before healing) and one after each consecutive healing event. In all curves the general trend is the same; the initially linear $P-d$ relation is followed by a visual deviation from linearity. In subsequent healing cycles, the sample exhibited a drop for the $P_{\max }$. Nevertheless the lowest value still remains well above that of the reference value (approximately $80 \%$ higher). In Figure 6a magnification of the curve at the crack initiation points is given and for each curve the points are marked. In the histogram of Figure $6 b$ the effect of the number of healing cycles on the mode II fracture characteristics $\left(P_{\max }\right.$ and $G_{\text {IIC }}$ ) of the hybrid CFRP is seen. As for the $P_{\max }$, the four healing cycles were able to reduce the recorded $P_{\max }$ value at a rate of approximately $33 \%$. After the first healing activation the $P_{\max }$ of the hybrid CFRP showed a decrease of approximately $16 \%$. The same behavior was
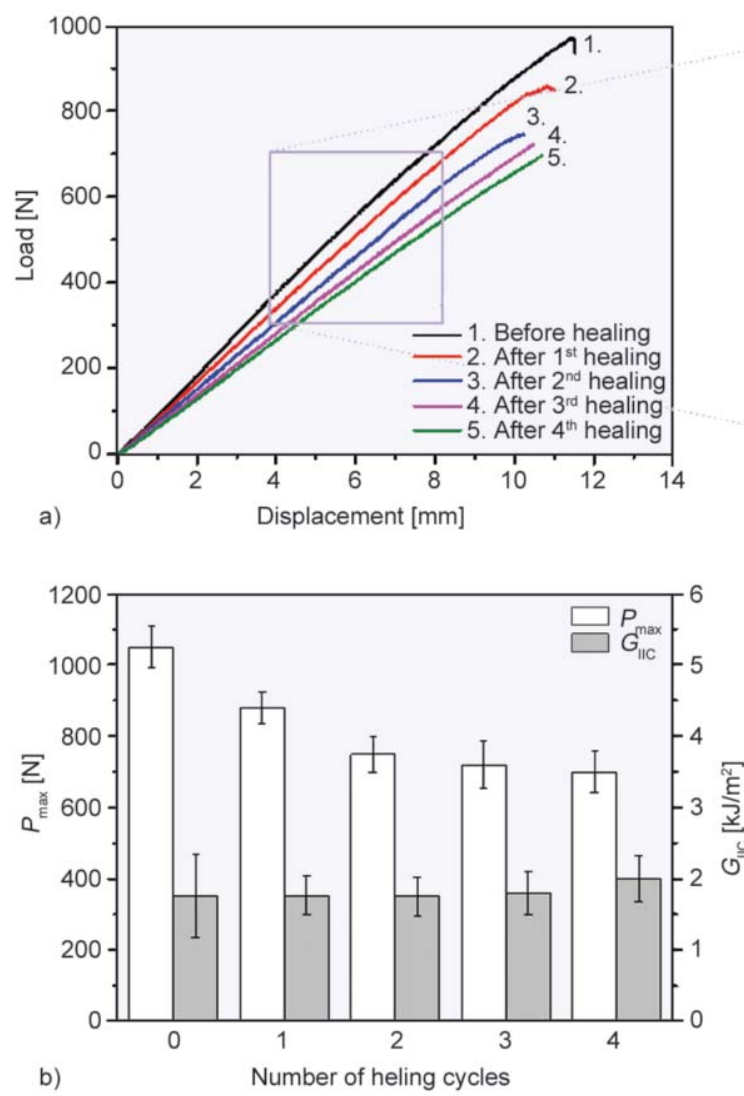

observed after the second healing cycle when compared to the first one. Following the third healing cycle the decrease of the $P_{\max }$ was calculated to be close to $4 \%$ comparing to the second one while the same decrease proportion was calculated after the fourth healing event. On the other hand the $G_{\text {IIC }}$ values exhibit a slight increase with increasing the number of healing activations; the $G_{\text {IIC }}$ value after the fourth healing cycle is $14 \%$ higher than that in the original (no-healed) situation. This behavior is attributed to the fact that the displacement value at crack propagation onset increases as the healing cycles increase (see Equation (1)). In Figure $6 \mathrm{c}$ the actual H.E. values for the two magnitudes of interest, calculated based on Equation (2), are given as a function of the number of healing cycles. In subsequent healing cycles a drop for the H.E. $P_{\max }$ is observed. Nevertheless the lowest value still remains above $70 \%$ of the reference value. On the other hand a slight total increase for the H.E.G GIC $_{\text {at }}$ at the rate of approximately
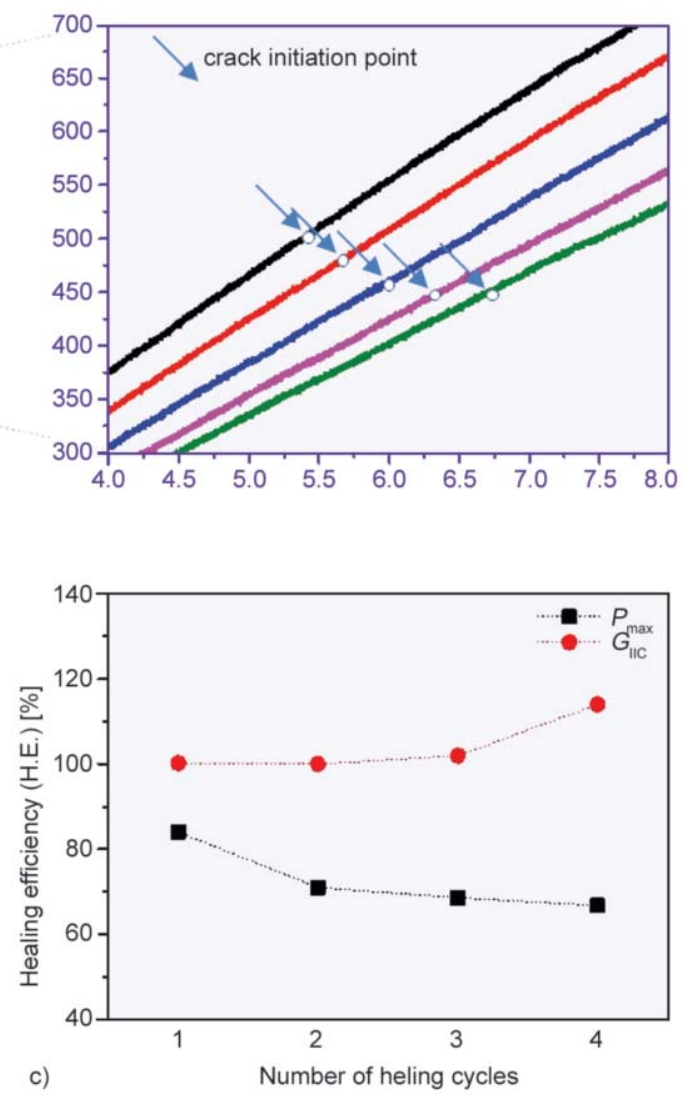

Figure 6. (a) Mode II interlaminar fracture toughness test: representative load-displacement curves for the hybrid CFRP before and after the healing cycles and determination of the crack initiation point. (b) Bar diagrams for the maximum load $\left(P_{\max }\right)$ and mode II interlaminar fracture toughness energy $\left(G_{\mathrm{IIC}}\right)$ values of the hybrid CFRP, before and after the healing cycles. (c) The effect of the number of healing cycles on the healing efficiency of the hybrid CFRP, in terms of the $P_{\max }$ and the $G_{\text {IIC. }}$. 
$7 \%$ is observed. The bending stiffness of the hybrid laminate presents a gradual decrease with increasing number of healing activations.

The repeatability of the healing effect is confirmed by the repetitive behavior of $\mathrm{AE}$ monitored during the healing cycles. This is more evident in the results of the bar chart in Figure 7 in which the cumulative $\mathrm{AE}$ hits and the cumulative AE energy values are depicted for both the crack initiation and fracture points. As clearly shown in this bar chart, the AE activity is reduced after the first healing cycle for both points and stayed rather stable after each consecutive healing cycle for the crack initiation point. For the fracture point a slight reduction of the AE characteristics after each healing cycle is the general trend whereas a slight increase was observed after the fourth healing cycle. The aforementioned behavior is a solid indication of the fact that the H.E. for the $P_{\max }$ value is reduced after each healing cycle. The ability of the SP material to partially remedy previously damaged sites in the vicinity of the crack path and thus produce new AE events (from the healed sites under re-loading) is finite and with a decreasing trend.

The partial recovery may is associated with the degradation of the epoxy-SP interface after each loading cycle which is only partially healed after the subsequent healing. Snapshots of the sample during the mode II experiments at the crack initiation and fracture points before and after the healing cycles are illustrated in Figure 8a.

The high H.E. proportions and the ability of the supramolecular interleaf to heal the cracks are attributed to the SP material's chains that have the ability

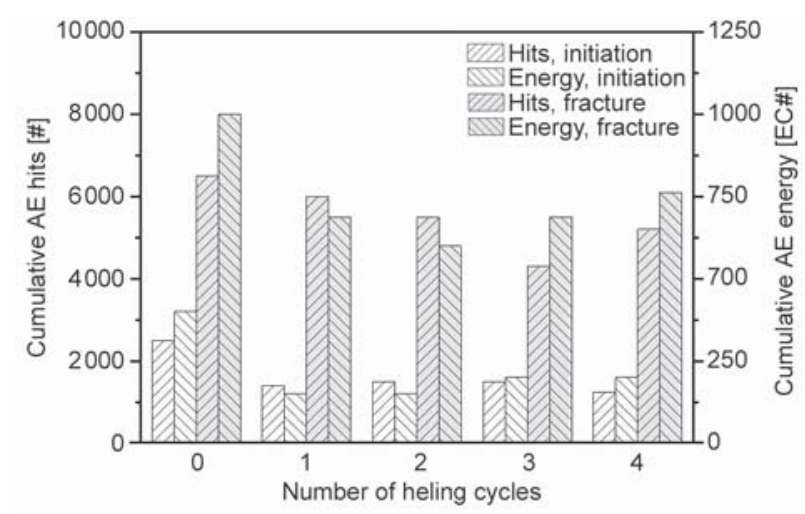

Figure 7. AE activity during mode II interlaminar fracture toughness tests of the hybrid CFRP at crack initiation and just before failure, before and after multiple healing cycles to reconnect themselves after the mechanical rapture during the mode II experiments. Critical parameters are a good coverage of the fractured surfaces by the SP material and a sufficiently low viscosity of the reversible polymer at $100^{\circ} \mathrm{C}$ in order to be able to flow into the crack flanks and to reposition itself equally over the debonded surface. Therefore, the observed reduction in the $P_{\max }$ value with increasing number of healing cycles can probably be attributed to an uneven spreading of the SP interleaf over the fracture surface during the healing cycle. An important point to note is the observation that the apparent flexural stiffness of the system decreases after multiple healing activations. A number of reasons have been identified to answer to this observation. Firstly, the tests extended to very large deformation which can lead to the breakage of the fibres on the outer layers. As a matter of fact, Figure $8 \mathrm{~b}$ shows evidence of broken fibres on the loading cross-head side. It is proposed that these excessive damages were reflected as portion reduction in the load vs. displacement curve. Secondly, during the healing agent activation it is possible that not the complete interface surface of the crack is recovered and bridged. Thus the system cannot recover the stiffness it formerly had. Finally, the healing functionality of the SP may degrade after multiple activations; an observation also noted in [29] with the same material. According to this work the bulk SP material is able to withstand at least seven healing cycles with excellent healing recoveries (still higher toughness values compared to the reference one), but in this loading case and because of the carbon fiber rupture the experiments were stopped after the fourth loading cycle. The posttesting examination of the fracture surface of the healed laminates revealed that the healing agent was clearly separated between the two adjacent fracture surfaces. Thus, the healing effect of the SP material was fully utilized.

\section{Conclusions}

In this work, the use of supramolecular polymers was investigated in the form of interleaves in CFRP as a means to integrate healing functionality to the composite. Mode II interlaminar fracture toughness tests were performed to assess the effects the SP material brings to the composite. It was shown that the SP interlayer which was introduced at the mid-plane of 


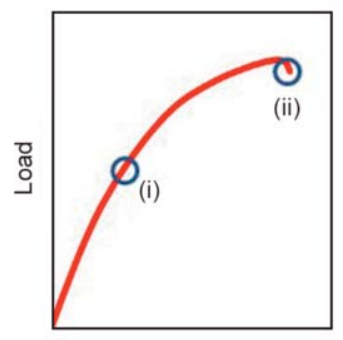

Displacement

a)

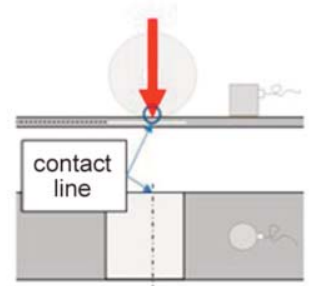

b)
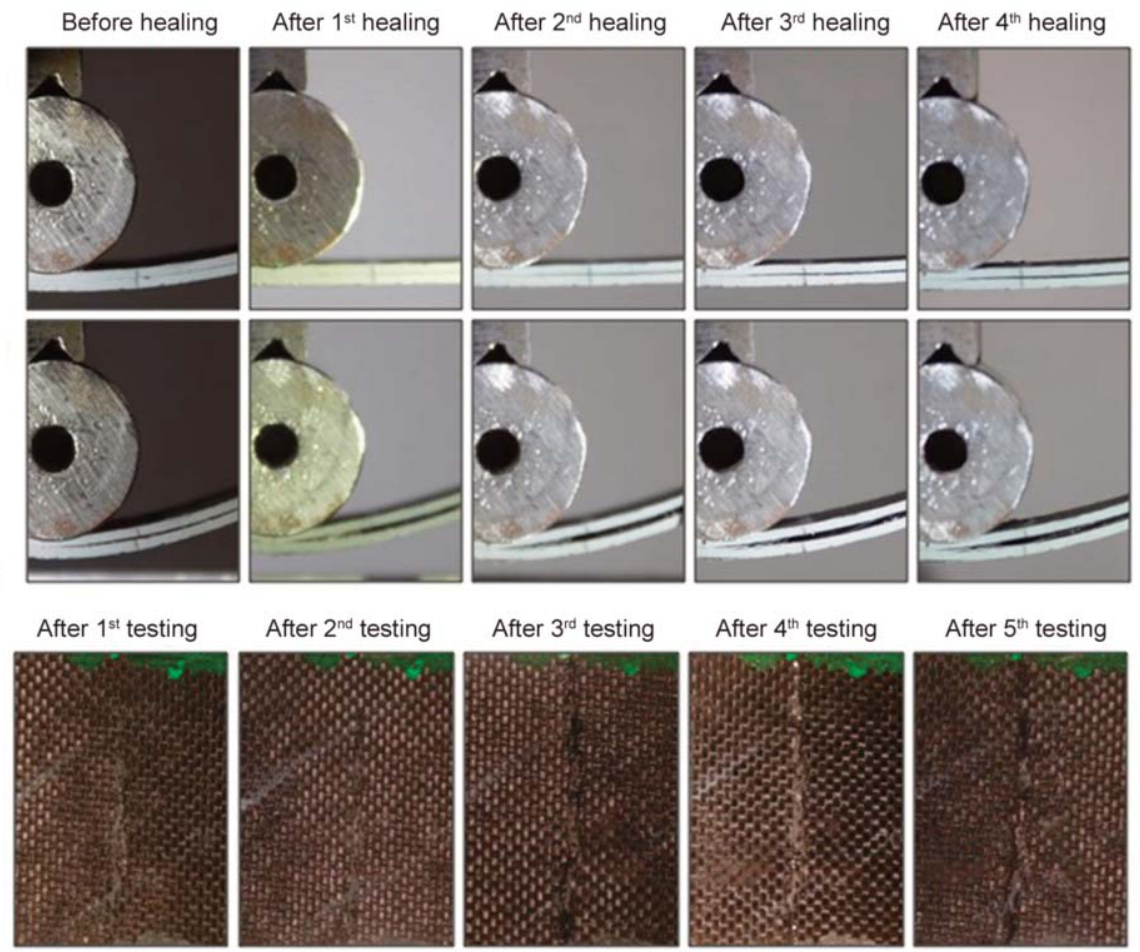

After $3^{\text {rd }}$ testing

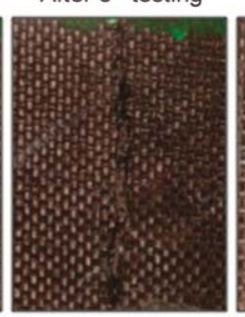

After $4^{\text {th }}$ testing

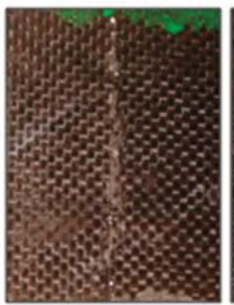

After $5^{\text {th }}$ testing

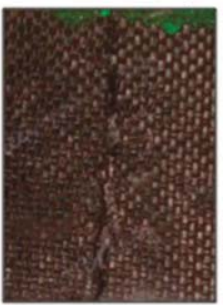

Figure 8. (a) Snapshot during the mode II interlaminar fracture toughness testing of the hybrid CFRP (i) just after crack propagation onset and (ii) just before failure (i.e. $P \sim P_{\max }$ ), before and after healing. (b) Photographs showing the progressive damage accumulation on the upper layers of the hybrid CFRP during the mode II testing, with increasing the number of testing cycles. The damaged line is the contact line between the specimen and the loading cylinder

the lay-up notably increased the fracture toughness of the hybrid composite system, when compared to the reference one. The $P_{\max }$ doubled while the $G_{\text {IIC }}$ multiplied two times. SP have incorporated a healing functionality to the composite. This has been justified by the effective recovery of more than $85 \%$ of the maximum load and $100 \%$ of the fracture toughness of the hybrid composite. A decreasing healing ability was observed in terms of load bearing capacity. AE has been utilized in-situ during the tests offering useful insights to the deformation and failure mechanisms taking place in the various materials involved i.e. hybrid, reference, before-after healing explaining inconsistencies or differences in their macroscopical behavior. These findings are considered very interesting for applications where damage tolerance is desired, such as in aeronautics. Such hybrid composite materials could dramatically decrease the repair down time of aircrafts.

\section{Acknowledgements}

The present work has been supported by the $7^{\text {th }}$ Framework Program of the European Commission under the project: Self-healing polymers for concepts on self-repaired aeronautical composites - HIPOCRATES (ACP3-GA-2013-605412).

\section{References}

[1] Yasaee M., Bond I. P., Trask R. S., Greenhalgh E. S.: Mode II interfacial toughening through discontinuous interleaves for damage suppression and control. Composites Part A: Applied Science and Manufacturing, 43, 121-128 (2012).

DOI: $10.1016 /$ j.compositesa.2011.09.026

[2] Ahmadi-Moghadam B., Taheri F.: Fracture and toughening mechanisms of GNP-based nanocomposites in modes I and II fracture. Engineering Fracture Mechanics, 131, 329-339 (2014).

DOI: $10.1016 /$ j.engfracmech.2014.08.008

[3] Zeng Y., Liu H-Y., Mai Y-W., Du X-S.: Effect of nanoparticle modification on composite mode II delamination. in ' $13^{\text {th }}$ International Conference on Fracture, Beijing, China’ p.9 (2013).

[4] Kostopoulos V., Karapappas P., Loutas T., Vavouliotis A., Paipetis A., Tsotra P.: Interlaminar fracture toughness of carbon fibre-reinforced polymer laminates with nano- and micro-fillers. Strain, 47, 269-282 (2011). DOI: 10.1111/j.1475-1305.2008.00612.x

[5] Mouritz A. P.: Review of z-pinned composite laminates. Composites Part A: Applied Science and Manufacturing, 38, 2383-2397 (2007). DOI: $\underline{10.1016 / \mathrm{j} . c o m p o s i t e s a .2007 .08 .016}$ 
[6] Magniez K., Chaffraix T., Fox B.: Toughening of a carbon-fibre composite using electrospun poly(hydroxyether of bisphenol A) nanofibrous membranes through inverse phase separation and inter-domain etherification. Materials, 4, 1967-1984 (2011).

DOI: $10.3390 / \mathrm{ma} 4111967$

[7] Aksoy A., Carlsson L. A.: Interlaminar shear fracture of interleaved graphite/epoxy composites. Composites Science and Technology, 43, 55-69 (1992). DOI: 10.1016/0266-3538(92)90133-N

[8] Molnár K., Koštáková E., Mészaros L.: The effect of needleless electrospun nanofibrous interleaves on mechanical properties of carbon fabrics/epoxy laminates. Express Polymer Letters, 8, 62-72 (2014). DOI: $10.3144 /$ expresspolymlett.2014.8

[9] Drakonakis V. M., Velisaris C. N., Seferis J. C., Doumanidis C. C., Wardle B. L., Papanicolaou G. C.: Matrix hybridization in the interlayer for carbon fiber reinforced composites. Polymer Composites, 31, 1965 1976 (2010). DOI: $10.1002 / p c .20996$

[10] Wu D. Y., Meure S., Solomon D.: Self-healing polymeric materials: A review of recent developments. Progress in Polymer Science, 33, 479-522 (2008). DOI: 10.1016/j.progpolymsci.2008.02.001

[11] White S. R., Sottos N. R., Geubelle P. H., Moore J. S., Kessler M. R., Sriram S. R., Brown E. N., Viswanathan S.: Autonomic healing of polymer composites. Nature, 409, 794-797 (2001). DOI: $10.1038 / 35057232$

[12] Diesendruck C. E., Sottos N. R., Moore J. S., White S. R.: Biomimetic self-healing. Angewandte Chemie International Edition, 127, 10572-10593 (2015). DOI: 10.1002/anie.201500484

[13] Hayes S. A., Zhang W., Branthwaite M., Jones F. R.: Self-healing of damage in fibre-reinforced polymer-matrix composites. Journal of the Royal Society, Interface, 4, 381-387 (2007). DOI: $10.1098 /$ rsif.2006.0209

[14] Pingkarawat K., Bhat T., Craze D. A., Wang C. H., Varley R. J., Mouritz A. P.: Healing of carbon fibre-epoxy composites using thermoplastic additives. Polymer Chemistry, 4, 5007-5015 (2015).

DOI: $10.1039 / \mathrm{C} 3 \mathrm{PY} 00459 \mathrm{G}$

[15] Wang C. H., Sidhu K., Yang T., Zhang J., Shanks R.: Interlayer self-healing and toughening of carbon fibre/ epoxy composites using copolymer films. Composites Part A: Applied Science and Manufacturing, 43, 512 518 (2012). DOI: 10.1016/j.compositesa.2011.11.020

[16] Yang T., Wang C. H., Zhang J., He S., Mouritz A. P.: Toughening and self-healing of epoxy matrix laminates using mendable polymer stitching. Composites Science and Technology, 72, 1396-1401 (2012). DOI: $\underline{10.1016 / \text { j.compscitech.2012.05.012 }}$
[17] Kotrotsos A., Baltopoulos A., Tsantzalis S., Tsilimigkra X., Tsokanas P., Kostopoulos V.: Experimental investigation on self-healing efficiency of doped fiber reinforced plastics with PET micro-particles. UPB Scientific Bulletin Series D: Mechanical Engineering, 78, 67-76 (2016).

[18] Brunsveld L., Folmer B. J. B., Meijer E. W., Sijbesma R. P.: Supramolecular polymers. Chemical Reviews, 101, 4071-4098 (2001). DOI: $10.1021 / \mathrm{cr} 990125 \mathrm{q}$

[19] Bosman A. W., Sijbesma R. P., Meijer E. W.: Supramolecular polymers at work. Materials Today, 7, 34-39 (2004).

DOI: $10.1016 / \mathrm{S} 1369-7021(04) 00187-7$

[20] de Greef T. F. A., Meijer E. W.: Materials science: Supramolecular polymers. Nature, 453, 171-173 (2008).

DOI: $10.1038 / 453171 \mathrm{a}$

[21] Palmer L. C., Velichko Y. S., de la Cruz M. O., Stupp S. I.: Supramolecular self-assembly codes for functional structures. Philosophical Transactions of the Royal Society A, 365, 1417-1433 (2007).

DOI: $10.1098 /$ rsta.2007.2024

[22] Stendahl J. C., Li L., Zubarev E. R., Chen Y-R., Stupp S. I.: Toughening of polymers by self-assembling molecules. Advanced Materials, 14, 1540-1543 (2002). DOI: 10.1002/1521-4095(20021104)14:21<1540::AIDADMA1540>3.0.CO;2-T

[23] Brunsveld L., Folmer B. J. B., Meijer E. W.: Supramolecular polymers. MRS Bulletin, 25, 49-53 (2000). DOI: $10.1557 / \mathrm{mrs} 2000.29$

[24] Cordier P., Tournilhac F., Soulié-Ziakovic C., Leibler L.: Self-healing and thermoreversible rubber from supramolecular assembly. Nature, 451, 977-980 (2008). DOI: 10.1038 /nature06669

[25] Sijbesma R. P., Beijer F. H., Brunsveld L., Folmer B. J. B., Hirschberg J. H. K. K., Lange R. F. M., Lowe J. K. L., Meijer E. W.: Reversible polymers formed from self-complementary monomers using quadruple hydrogen bonding. Science, 278, 1601-1604 (1997). DOI: $10.1126 /$ science. 278.5343 .1601

[26] Söntjens S. H. M., Renken R. E. R., van Gemert G. M. L., Engels T. A. P., Bosman W. A., Janssen H. M., Govaert L. E., Baaijens F. P. T.: Thermoplastic elastomers based on strong and well-defined hydrogen-bonding interactions. Macromolecules, 41, 5703-5708 (2008). DOI: $10.1021 / \mathrm{ma} 800744 \mathrm{c}$

[27] Monemian S., Korley L. T. J.: Exploring the role of supramolecular associations in mechanical toughening of interpenetrating polymer networks. Macromolecules, 48, 7146-7155 (2015).

DOI: 10.1021/acs.macromol.5b01752

[28] Zhang L., Brostowitz N. R., Cavicchi K. A., Weiss R. A.: Perspective: Ionomer research and applications. Macromolecular Reaction Engineering, 8, 81-99 (2014). DOI: $\underline{10.1002 / \text { mren.201300181 }}$ 
[29] Kostopoulos V., Kotrotsos A., Tsantzalis S., Tsokanas P., Loutas T., Bosman A.W.: Toughening and healing of continuous fibre reinforced composites by supramolecular polymers. Composites Science and Technology, 128, 84-93 (2016).

DOI: $10.1016 /$ j.compscitech.2016.03.021

[30] AITM 1.0005: Carbon fiber reinforced plastics. Determination of interlaminar fracture toughness energy. Mode I (1994).

[31] AITM 1.0006: Carbon fiber reinforced plastics. Determination of interlaminar fracture toughness energy. Mode II (1994).
[32] Maples H. A., Wakefield S., Robinson P., Bismarck A.: High performance carbon fibre reinforced epoxy composites with controllable stiffness. Composites Science and Technology, 105, 134-143 (2014).

DOI: 10.1016/j.compscitech.2014.09.008

[33] Groleau M. R., Shi Y-B., Yee A. F., Bertram J. L., Sue H. J., Yang P. C.: Mode II fracture of composites interlayered with nylon particles. Composites Science and Technology, 56, 1223-1240 (1996). DOI: $10.1016 / \mathrm{S} 0266-3538(96) 00080-2$

[34] van Gemert G. M. L., Peeters J. W., Söntjens S. H. M., Janssen H. M., Bosman A. W.: Self-healing supramolecular polymers in action. Macromolecular Chemistry and Physics, 213, 234-242 (2012).

DOI: $\underline{10.1002 / \mathrm{macp} .201100559}$ 